WojCiech Zieliński (Warszawa)

\title{
THE SHORTEST CONFIDENCE INTERVAL FOR PROPORTION IN FINITE POPULATIONS
}

Abstract. Consider a finite population. Let $\theta \in(0,1)$ denote the proportion of units with a given property. The problem is to estimate $\theta$ on the basis of a sample drawn according to simple random sampling without replacement. We are interested in interval estimation of $\theta$. We construct the shortest confidence interval at a given confidence level.

Introduction. The problem of the interval estimation of the proportion (fraction) $\theta$ is very old. The first solution was given by Clopper and Pearson (1934) and since then many authors have dealt with the problem. Zielinski (2010a, 2012, 2016) considered the problem of constructing the shortest confidence intervals for probability of success in a binomial as well as in a negative binomial model. The solutions are valid for infinite populations.

In many applications (economic, social, etc.) we deal with a finite population, so we are interested in interval estimation of $\theta$ in that case. Remarks on differences in statistical inference in infinite and finite populations may be found for example in Yates (1960) or Cochran (1977). The problem of interval estimation of proportion in finite populations was rather rarely considered in literature. This is because the construction of the exact confidence interval is based on the hypergeometric distribution. Different approximations may be found in Buonaccorsi (1987), Wendell and Schmee (2001) or Wright (1991). There are at least two approximations commonly used in applications: binomial and normal. However, using those approximations may lead to wrong conclusions (Zieliński 2011). In what follows, the exact (hypergeometric) distribution will be used.

2010 Mathematics Subject Classification: Primary 62F25; Secondary 62D99.

Key words and phrases: proportion, confidence interval, shortest confidence intervals, finite population.

Received 22 February 2016; revised 30 June 2016.

Published online 30 September 2016. 
Confidence interval. Consider a population $\mathcal{U}=\left\{u_{1}, \ldots, u_{N}\right\}$ containing the finite number $N$ of units. Let $M$ denote the unknown number of objects in the population which have an interesting property. We are interested in interval estimation of $M$, or equivalently, of the fraction $\theta=M / N$. A sample of size $n$ is drawn according to simple random sampling without replacement. Let $\xi$ be the random variable describing the number of objects with the given property in the sample. On the basis of $\xi$ we want to construct a confidence interval for $\theta$ at a confidence level $\delta$.

The random variable $\xi$ has the hypergeometric distribution (Johnson and Kotz 1969, Zieliński 2010b)

$$
P_{\theta, N, n}\{\xi=x\}=\frac{\left(\begin{array}{c}
\theta N \\
x
\end{array}\right)\left(\begin{array}{c}
(1-\theta) N \\
n-x
\end{array}\right)}{\left(\begin{array}{c}
N \\
n
\end{array}\right)},
$$

for integers $x$ from the interval $[\max \{0, n-(1-\theta) N\}, \min \{n, \theta N\}]$. Let $f_{\theta, N, n}(\cdot)$ be the probability distribution function, i.e.

$f_{\theta, N, n}(x)$

$$
= \begin{cases}P_{\theta, N, n}\{\xi=x\} & \text { for integer } x \in[\max \{0, n-(1-\theta) N\}, \min \{n, \theta N\}], \\ 0 & \text { elsewhere, }\end{cases}
$$

and let

$$
F_{\theta, N, n}(x)=\sum_{t \leq x} f_{\theta, N, n}(t)
$$

be the cumulative distribution function (CDF) of $\xi$. Then

$$
\begin{aligned}
F_{\theta, N, n}(x)= & \frac{\Gamma(\theta N+1) \Gamma((1-\theta) N+1)}{n !\left(\begin{array}{c}
N \\
n
\end{array}\right)} \\
& \cdot \sum_{t \leq x}\left(\begin{array}{c}
n \\
t
\end{array}\right) \frac{1}{\Gamma(\theta N-t+1) \Gamma((1-\theta) N-n+t+1)} \\
= & 1-\frac{\left(\begin{array}{c}
n \\
x+1
\end{array}\right)\left(\begin{array}{c}
N-n \\
\theta N-x-1
\end{array}\right)}{\left(\begin{array}{c}
N \\
\theta N
\end{array}\right)} \\
& \cdot{ }_{3} F_{2}[\{1, x+1-\theta N, x+1-n\},\{x+2,(1-\theta) N+x+2-n\} ; 1],
\end{aligned}
$$

where

$$
{ }_{3} F_{2}\left[\left\{a_{1}, a_{2}, a_{3}\right\},\left\{b_{1}, b_{2}\right\} ; t\right]=\sum_{k=0}^{\infty} \frac{\left(a_{1}\right)_{k}\left(a_{2}\right)_{k}\left(a_{3}\right)_{k}}{\left(b_{1}\right)_{k}\left(b_{2}\right)_{k}} \frac{t^{k}}{k !}
$$

and $(a)_{k}=a(a+1) \cdots(a+k-1)$.

The construction of the confidence interval at a confidence level $\delta$ for $\theta$ is based on this cumulative distribution function. Let $\theta(x, N, n, \alpha)$ be the solution of the equation $F_{\theta, N, n}(x)=\alpha$ with respect to $\theta$. If $\xi=x$ is observed 
then the confidence interval has the form

$$
\left(\theta_{L}\left(x-1, N, n, \delta_{1}\right), \theta_{U}\left(x, N, n, \delta_{2}\right)\right) .
$$

The numbers $\delta_{1}$ and $\delta_{2}$ are such that $\delta_{1}-\delta_{2}=\delta$. For $\xi=0$ the left end is taken to be 0 , and for $\xi=n$ the right end is taken to be 1 . The analytic solution is unavailable. However, for given $x, n$ and $N$, the confidence interval may be found numerically.

To be more precise, the confidence interval for $\theta$ is in fact a confidence set of the numbers of the form $k / N$ between $\left\lfloor N \cdot \theta_{L}\left(x-1, N, n, \delta_{1}\right)\right\rfloor / N$ and $\left\lfloor N \cdot \theta_{U}\left(x, N, n, \delta_{1}\right)+1\right\rfloor / N$ (here $\lfloor t\rfloor$ denotes the greatest integer not greater than $t)$. In what follows, we will make use of $(*)$.

In the standard construction $\delta_{1}=(1+\delta) / 2$ is used. It is of interest to find the shortest confidence interval. Therefore, we want to find $\delta_{1}$ and $\delta_{2}$ such that the confidence interval is the shortest possible.

The shortest confidence interval. Consider the length of the confidence interval when $\xi=x$ is observed:

$$
d\left(\delta_{1}, x\right)=\theta_{U}\left(x, N, n, \delta_{1}\right)-\theta_{L}\left(x-1, N, n, \delta+\delta_{1}\right) .
$$

In what follows we consider only the case $x \leq n / 2$. If $x \geq n / 2$, the role of success and failure should be interchanged.

Let us state the following theorem.

THEOREM 1. For $x>1$ there exists a two-sided shortest confidence interval. For $x=1$ the shortest confidence interval is one-sided.

To prove the theorem we will prove the following lemma. Define $G_{x, N, n}(\theta)$ $=F_{\theta, N, n}(x)$. Of course, the domain of this function is $[x / N, 1-(n-x) / N]$.

Lemma. For $x \geq 1$ there exists $\theta^{*}$ such that the function $G_{x, N, n}(\cdot)$ is concave for $\theta<\theta^{*}$, and convex for $\theta>\theta^{*}$.

Proof. Consider the second order differences of the function $G_{x, N, n}(\cdot)$. Some calculations give:

$$
\begin{array}{r}
H_{x, N, n}(\theta) \\
=\left(G_{x, N, n}\left(\theta+\frac{1}{N}\right)-G_{x, N, n}(\theta)\right)-\left(G_{x, N, n}(\theta)-G_{x, N, n}\left(\theta-\frac{1}{N}\right)\right) \\
=\frac{1}{\left(\begin{array}{c}
N \\
n
\end{array}\right)} \sum_{t=0}^{x} \frac{(N+1) t(t-1)-(n-1)(2 t(N+1)-n) \theta+N n(n-1) \theta^{2}}{(1-\theta) \theta N(\theta N+1-t)((1-\theta) N+1-(n-t))} \\
\cdot\left(\begin{array}{c}
\theta N \\
t
\end{array}\right)\left(\begin{array}{c}
(1-\theta) N \\
n-t
\end{array}\right) .
\end{array}
$$

Because

$$
H_{x, N, n}\left(\frac{x}{N}\right)=-\frac{n !(N-x-1) !}{N !(n-x-1) !}<0
$$


and

$$
\begin{aligned}
H_{x, N, n}(1 & \left.-\frac{n-x}{N}\right) \\
& =\frac{n !(N-n+x-1) !}{N ! x !}(N(n-x+1)-(n-1)(n-x))>0
\end{aligned}
$$

the equation

$$
H_{x, N, n}(\theta)=0
$$

has a solution.

The hypergeometric distributions are stochastically ordered, i.e. for $\theta_{1}<\theta_{2}$ and for any given $x$ we have $F_{\theta_{1}, N, n}(x)>F_{\theta_{2}, N, n}(x)$. Hence, the equation $H_{x, N, n}(\theta)=0$ has only one solution $\theta^{*}$. We have

$$
H_{x, N, n}(\theta) \begin{cases}<0 & \text { for } \theta<\theta^{*}, \\ >0 & \text { for } \theta>\theta^{*} .\end{cases}
$$

Hence $G_{x, N, n}(\cdot)$ is concave for $\theta<\theta^{*}$ and convex for $\theta>\theta^{*}$.

Proof of Theorem 1. We have to show that for $x>1$ there exists $0<\delta_{1}<1-\delta$ such that $d\left(\delta_{1}, x\right)$ is minimal. The length of the confidence interval equals

$$
d\left(\delta_{1}, x\right)=\theta_{U}\left(x, N, n, \delta_{1}\right)-\theta_{L}\left(x-1, N, n, \delta_{1}+\delta\right),
$$

where $\theta_{U}\left(x, N, n, \delta_{1}\right)$ is a solution of $F_{\theta_{U}, N, n}(x)=\delta_{1}$ and $\theta_{L}\left(x-1, N, n, \delta_{1}+\delta\right)$ is a solution of $F_{\theta_{L}, N, n}(x-1)=\delta_{1}+\delta$. By Lemma, for $x>1$ the function $(0,1-\delta) \ni \delta_{1} \mapsto \theta_{U}\left(x, N, n, \delta_{1}\right)$ is convex and $(0,1-\delta) \ni \delta_{1} \mapsto \theta_{L}(x-1$, $\left.N, n, \delta_{1}+\delta\right)$ is concave. Hence, there exists $\delta_{1}^{*}$ such that

$$
d\left(\delta_{1}^{*}, x\right)=\inf \left\{d\left(\delta_{1}, x\right): \delta<\delta_{1}<1\right\}
$$

Consider now the case $x=1$. The lower bound of the confidence interval is a solution of $F_{\theta_{L}, N, n}(0)=\delta_{1}+\delta$. It is easy to check that $H_{0, N, n}(\theta)>0$ for all $\theta \in[1 / N, 1-(n-1) / N]$. Hence the function $(0,1-\delta) \ni \delta_{1} \mapsto$ $\theta_{L}\left(0, N, n, \delta_{1}+\delta\right)$ is concave. The length of the confidence interval reaches its minimum at $\delta_{1}=1-\delta$, so the shortest confidence interval for $x=1$ is one-sided.

It is interesting to note that if $n$ is even and $x=n / 2$ then the shortest confidence interval is the standard one. This follows from the fact that for $\theta<0.5$

$$
G_{n / 2, N, n}(\theta)=G_{n / 2, N, n}(1-\theta) .
$$

In Tables 1 and 2 some numerical results are given. The standard confidence intervals $\left(\theta_{L}^{\mathrm{st}}, \theta_{U}^{\mathrm{st}}\right)$ and the shortest confidence intervals $\left(\theta_{L}^{\mathrm{sh}}, \theta_{U}^{\mathrm{sh}}\right)$ for 
Table 1. The shortest confidence intervals $(n=10)$

\begin{tabular}{rcrccr}
\hline$x$ & $\theta_{L}^{\text {st }}$ & $\theta_{U}^{\text {st }}$ & $\theta_{L}^{\text {sh }}$ & $\theta_{U}^{\text {sh }}$ & \\
\hline 0 & 0.000000 & 0.307106 & 0.000000 & 0.257698 & $83.91 \%$ \\
1 & 0.002517 & 0.443510 & 0.000000 & 0.392886 & $89.09 \%$ \\
2 & 0.025602 & 0.554589 & 0.007924 & 0.509322 & $94.78 \%$ \\
3 & 0.067444 & 0.651012 & 0.048707 & 0.621332 & $98.12 \%$ \\
4 & 0.122510 & 0.736298 & 0.111168 & 0.722398 & $99.58 \%$ \\
5 & 0.188249 & 0.811751 & 0.188249 & 0.811751 & $100.00 \%$ \\
6 & 0.263702 & 0.877490 & 0.277602 & 0.888832 & $99.58 \%$ \\
7 & 0.348988 & 0.932556 & 0.378668 & 0.951293 & $98.12 \%$ \\
8 & 0.445411 & 0.974398 & 0.490678 & 0.992076 & $94.78 \%$ \\
9 & 0.556490 & 0.997483 & 0.607114 & 1.000000 & $89.09 \%$ \\
10 & 0.692894 & 1.000000 & 0.742302 & 1.000000 & $83.91 \%$ \\
\hline
\end{tabular}

Table 2. The shortest confidence intervals $(n=100)$

\begin{tabular}{rccccr}
\hline$x$ & $\theta_{L}^{\text {st }}$ & $\theta_{U}^{\text {st }}$ & $\theta_{L}^{\text {sh }}$ & $\theta_{U}^{\text {sh }}$ & \\
\hline 0 & 0.000000 & 0.034392 & 0.000000 & 0.028026 & $81.49 \%$ \\
1 & 0.000240 & 0.052222 & 0.000000 & 0.044721 & $86.03 \%$ \\
2 & 0.002867 & 0.067851 & 0.001000 & 0.059529 & $90.07 \%$ \\
3 & 0.006981 & 0.082404 & 0.003940 & 0.074355 & $93.36 \%$ \\
4 & 0.012023 & 0.096283 & 0.008441 & 0.088570 & $95.10 \%$ \\
5 & 0.017685 & 0.109684 & 0.013809 & 0.102289 & $96.17 \%$ \\
6 & 0.023799 & 0.122721 & 0.019758 & 0.115618 & $96.90 \%$ \\
7 & 0.030264 & 0.135469 & 0.026131 & 0.128634 & $97.43 \%$ \\
8 & 0.037008 & 0.147978 & 0.032833 & 0.141392 & $97.83 \%$ \\
9 & 0.043986 & 0.160284 & 0.039800 & 0.153931 & $98.14 \%$ \\
10 & 0.051163 & 0.172415 & 0.046991 & 0.166283 & $98.38 \%$ \\
15 & 0.089272 & 0.231062 & 0.085363 & 0.225911 & $99.12 \%$ \\
20 & 0.129994 & 0.287288 & 0.126509 & 0.282997 & $99.49 \%$ \\
25 & 0.172541 & 0.341782 & 0.169557 & 0.338284 & $99.70 \%$ \\
30 & 0.216514 & 0.394901 & 0.214077 & 0.392154 & $99.83 \%$ \\
35 & 0.261684 & 0.446856 & 0.259824 & 0.444828 & $99.91 \%$ \\
40 & 0.307912 & 0.497773 & 0.306651 & 0.496440 & $99.96 \%$ \\
45 & 0.355111 & 0.547728 & 0.354472 & 0.547071 & $99.99 \%$ \\
50 & 0.403237 & 0.596763 & 0.403237 & 0.596763 & $100.00 \%$ \\
\hline
\end{tabular}

$N=1000$ and $\delta=0.95$ are shown. In the last column the ratio of the length of the shortest to the length of the standard confidence interval is calculated.

In Figure 1 the confidence level of the shortest confidence interval is shown $(N=1000, n=100, \delta=0.95)$. Note that, for some $\theta$, the confidence level is smaller than the nominal one. This is in contradiction to the definition of the confidence interval (Neyman 1934, Cramér 1946, Lehmann 1959, Silvey 1970). In what follows, a small modification is introduced, after which the shortest confidence interval does not have this disadvantage. 


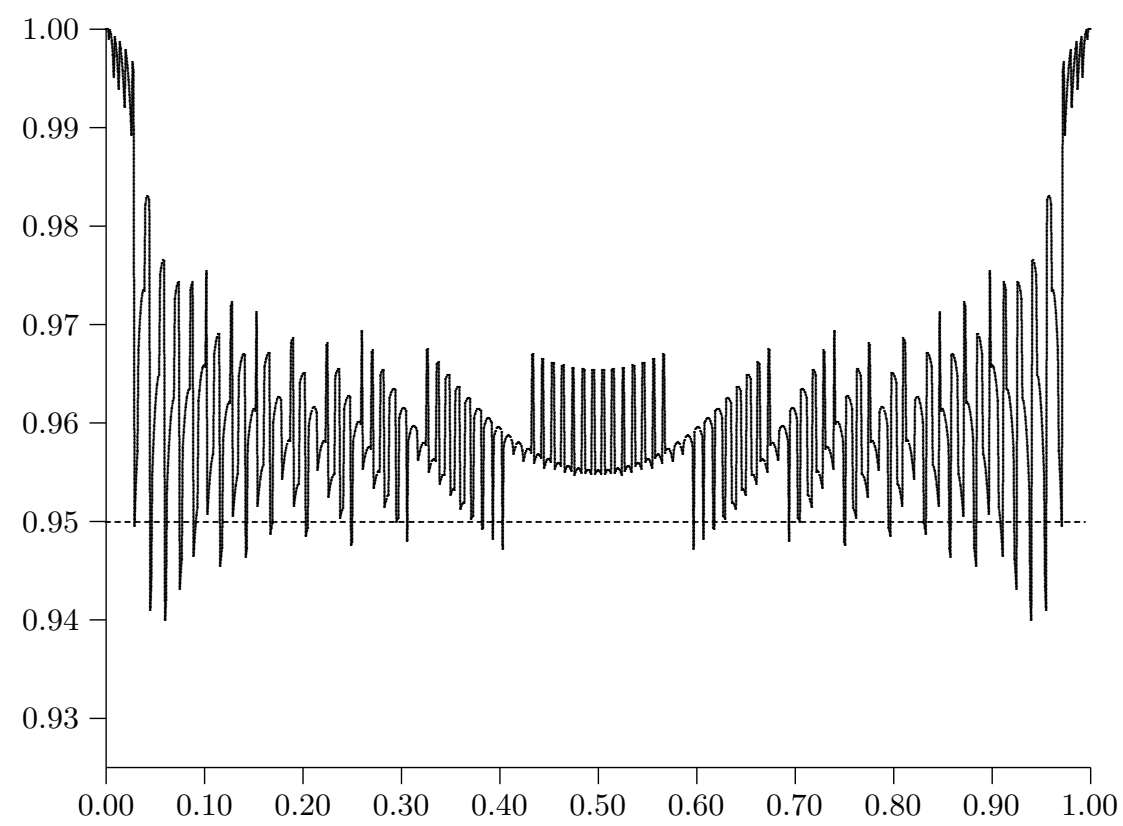

Fig. 1. Confidence level of the shortest confidence interval

Auxiliary variable. Let $\eta$ be a random variable conditionally distributed on the interval $[0,1]$ with $\operatorname{CDF} G_{\eta \mid \xi=x}(\cdot)$. The confidence interval will be constructed on the basis of $\zeta=\xi+\eta$. The distribution of that r.v. is easy to obtain: $P_{\theta, N, n}\{\zeta \leq t\}$

$$
= \begin{cases}0 & \text { if } t \leq 0, \\ \alpha(\lfloor t\rfloor,\{t\}) P_{\theta, N, n}\{\xi=\lfloor t\rfloor\} & \text { if }\lfloor t\rfloor=0, \\ \sum_{k=0}^{\lfloor t\rfloor-1} P_{\theta, N, n}\{\xi=k\}+\alpha(\lfloor t\rfloor,\{t\}) P_{\theta, N, n}\{\xi=\lfloor t\rfloor\} & \text { if } 1 \leq\lfloor t\rfloor \leq n, \\ 1 & \text { if }\lfloor t\rfloor>n,\end{cases}
$$

where

$$
\{t\}=t-\lfloor t\rfloor \quad \text { and } \quad \alpha(\lfloor t\rfloor,\{t\})=\int_{0}^{\{t\}} G_{\eta \mid \xi=\lfloor t\rfloor}(d u) .
$$

It is easy to note that the distribution of $\eta$ may be taken to be uniform $U(0,1)$ independently of $\xi$. Let

$$
\mathcal{G}_{x, y, N, n}(\theta)=(1-y) F_{\theta, N, n}(x-1)+y F_{\theta, N, n}(x) .
$$

Then

$$
\theta_{L}\left(x, y, N, n, \delta_{1}+\delta\right)=\mathcal{G}_{x, y, N, n}^{-1}\left(\delta_{1}+\delta\right)
$$

and

$$
\theta_{U}\left(x, y, N, n, \delta_{1}\right)=\mathcal{G}_{x+1, y, N, n}^{-1}\left(\delta_{1}\right)
$$


are the ends of the confidence interval for $\theta$ at the confidence level $\delta$. The length of the confidence interval equals

$$
d\left(\delta_{1} ; x, y\right)=\mathcal{G}_{x+1, y, N, n}^{-1}\left(\delta_{1}\right)-\mathcal{G}_{x, y, N, n}^{-1}\left(\delta_{1}+\delta\right) .
$$

Let $\xi=x$ and $\eta=y$ be observed. We are looking for $\delta_{1}^{*}$ such that

$$
d\left(\delta_{1}^{*} ; x, y\right)=\min \left\{d\left(\delta_{1} ; x, y\right): 0 \leq \delta_{1} \leq 1-\delta\right\} .
$$

TheOREm 2. If $x \geq 2$ then the shortest confidence interval is two-sided.

Proof. Consider the second order differences

$$
\begin{aligned}
& \mathcal{H}_{x, y, N, n}(\theta) \\
& \quad=\left(\mathcal{G}_{x, y, N, n}\left(\theta+\frac{1}{N}\right)-\mathcal{G}_{x, y, N, n}(\theta)\right)-\left(\mathcal{G}_{x, y, N, n}(\theta)-\mathcal{G}_{x, y, N, n}\left(\theta-\frac{1}{N}\right)\right) \\
& =(1-y) H_{x, N, n}(\theta)+y H_{x+1, N, n}(\theta) .
\end{aligned}
$$

Analysis similar to the one in the proof of the Lemma shows that for $x \geq 2$ and $y \in(0,1)$,

$$
\mathcal{H}_{x, y, N, n}\left(\frac{x}{N}\right)<0 \quad \text { and } \quad \mathcal{H}_{x, y, N, n}\left(1-\frac{n-x}{N}\right)>0 .
$$

Hence there exists a two-sided shortest confidence interval.

TheOREm 3. For $x=1$ there exists $y^{*}$ such that the shortest confidence interval is one-sided if $x+y<1+y^{*}$, and two-sided otherwise.

Proof. For convenience assume that $\theta$ is a continuous parameter. The derivative of $d\left(\delta_{1} ; x, y\right)$ with respect to $\delta_{1}$ equals

$$
\frac{\partial d\left(\delta_{1} ; x, y\right)}{\partial \delta_{1}}=\frac{1}{\operatorname{LHS}\left(\delta_{1} ; x, y\right)}-\frac{1}{\operatorname{RHS}\left(\delta_{1} ; x, y\right)},
$$

where

$$
\begin{aligned}
\operatorname{LHS}\left(\delta_{1} ; x, y\right) & =\left.\frac{\partial \mathcal{G}_{x+1, y, N, n}(\theta)}{\partial \theta}\right|_{\theta=\mathcal{G}_{x+1, y, N, n}^{-1}\left(\delta_{1}\right)}, \\
\operatorname{RHS}\left(\delta_{1} ; x, y\right) & =\left.\frac{\partial \mathcal{G}_{x, y, N, n}(\theta)}{\partial \theta}\right|_{\theta=\mathcal{G}_{x, y, N, n}^{-1}\left(\delta_{1}+\delta\right)} .
\end{aligned}
$$

We have

$$
\begin{aligned}
& \frac{\partial \mathcal{G}_{1, y, N, n}(\theta)}{\partial \theta}=\frac{N \Gamma((1-\theta) N+1)}{\left(\begin{array}{l}
N \\
n
\end{array}\right) \Gamma(n+1) \Gamma((1-\theta) N+1-n)} \\
& \cdot[n y-1-(N+1-n+N \theta(n y-1))(H((1-\theta) N)-H((1-\theta) N-n))]
\end{aligned}
$$


and

$$
\begin{aligned}
& \frac{\partial \mathcal{G}_{2, y, N, n}(\theta)}{\partial \theta}=\frac{N \Gamma((1-\theta) N+1)}{2\left(\begin{array}{l}
N \\
n
\end{array}\right) \Gamma(n+1) \Gamma((1-\theta) N+3-n)} \\
& \cdot[2(n-1)(N+2-n)-2(N+1-n)+n(n-1) y \\
& -(N(n-1) n y \theta(\theta N-1)+2((1-\theta) N+2-n)(N+1-n+N(n-1) \theta)) \\
& \cdot(H((1-\theta) N)-H((1-\theta) N+2-n))],
\end{aligned}
$$

where $H(u)=\sum_{i=1}^{u} 1 / i$.

If $\delta_{1} \rightarrow 0$, then

$$
\mathcal{G}_{1, y, N, n}^{-1}\left(\delta_{1}\right) \rightarrow \mathcal{G}_{1, y, N, n}^{-1}(0)=1
$$

and

$$
\mathcal{G}_{2, y, N, n}^{-1}\left(\delta_{1}+\delta\right) \rightarrow \mathcal{G}_{2, y, N, n}^{-1}(\delta)>0
$$

and for all $y$,

$$
\operatorname{RHS}\left(\delta_{1} ; 1, y\right) \rightarrow \infty \text { and } \operatorname{LHS}\left(\delta_{1} ; 1, y\right) \rightarrow \operatorname{LHS}(0 ; 1,0)<0
$$

Hence $\frac{\partial d\left(\delta_{1} ; 1, y\right)}{\partial \delta_{1}}<0$ as $\delta_{1} \rightarrow 0$.

If $\delta_{1} \rightarrow 1-\delta$, then

$$
\mathcal{G}_{1, y, N, n}^{-1}\left(\delta_{1}\right) \rightarrow \mathcal{G}_{1, y, N, n}^{-1}(1-\delta)>0
$$

and

$$
\mathcal{G}_{2, y, N, n}^{-1}\left(\delta_{1}+\delta\right) \rightarrow \mathcal{G}_{2, y, N, n}^{-1}(1)=1 / N
$$

Since $\operatorname{RHS}\left(1-\delta_{1} ; 1,0\right)<0<\operatorname{LHS}\left(1-\delta_{1} ; 1,0\right)\left(\right.$ so $\left.\left.\frac{\partial d\left(\delta_{1} ; 1,1\right)}{\partial \delta_{1}}\right|_{\delta_{1}=0}>0\right)$ and $\operatorname{RHS}\left(1-\delta_{1} ; 1,1\right)<\operatorname{LHS}\left(1-\delta_{1} ; 1,1\right)<0\left(\right.$ so $\left.\left.\frac{\partial d\left(\delta_{1} ; 1,0\right)}{\partial \delta_{1}}\right|_{\delta_{1}=0}<0\right)$, there exists $y^{*}$ such that $\operatorname{LHS}\left(1-\delta_{1} ; 1, y^{*}\right)=\operatorname{RHS}\left(1-\delta_{1} ; 1, y^{*}\right)$. So, the shortest confidence interval is one-sided for $y<y^{*}$, and two-sided otherwise.

In Table 3 the values of $y^{*}$ for different population sizes $N$ and sample sizes $n(1 \%, 5 \%$ and $10 \%$ of population size) are given.

Table 3. Values of $y^{*}$

\begin{tabular}{rccc}
\hline$N$ & $1 \%$ & $5 \%$ & $10 \%$ \\
\hline 1000 & 0.99466 & 0.96762 & 0.93372 \\
5000 & 0.99358 & 0.96652 & 0.93202 \\
10000 & 0.99345 & 0.96638 & 0.93181 \\
\hline
\end{tabular}

In Figure 2 the confidence level of the randomized shortest confidence interval for $N=1000, n=100$ and $\delta=0.95$ is shown.

Final remarks. 1. The above considerations may be summarized as follows. From a finite population of size $N$ we draw a sample of size $n$ (according to simple random sampling without replacement). Observe the r.v. $\xi$ with 


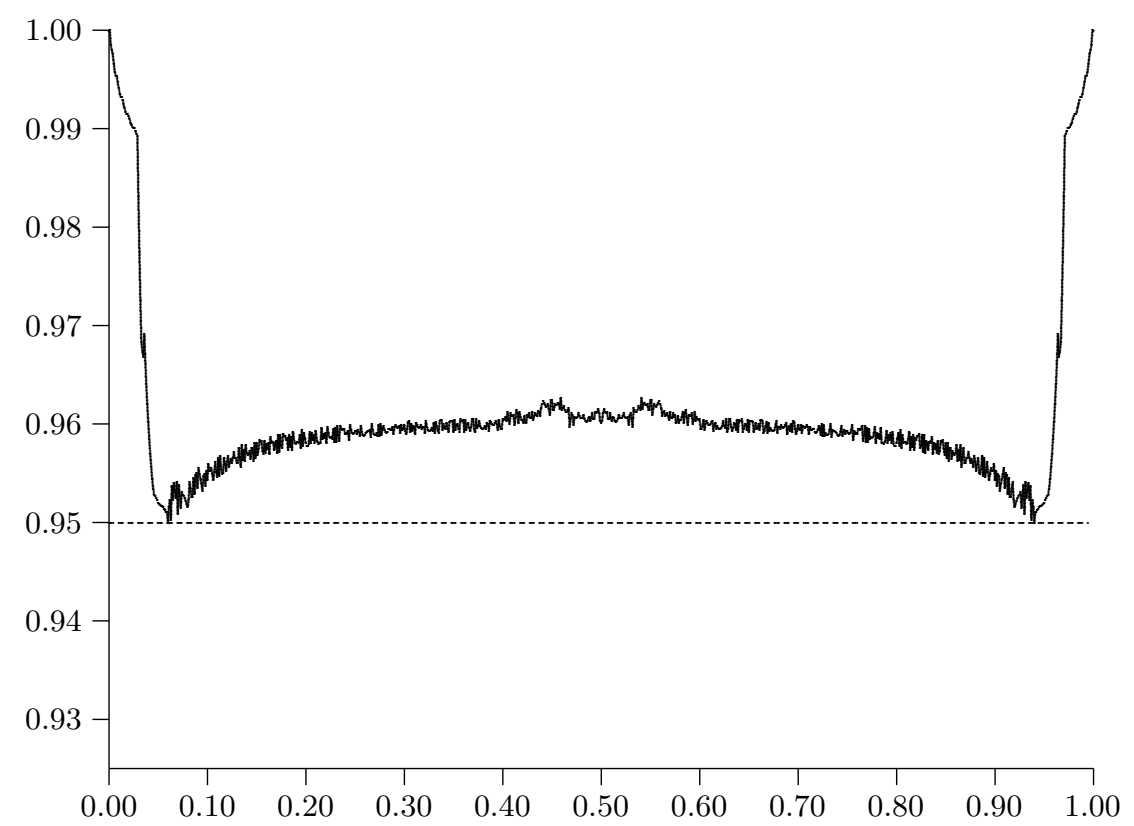

Fig. 2. Confidence level of the randomized shortest confidence interval

hypergeometric distribution with parameters $\theta, N, n$. Draw a r.v. $\eta$ according to $U(0,1)$ distribution. For $\xi \leq n / 2$ consider $\xi+\eta$. The shortest confidence interval for $\theta$ is one-sided if $\xi+\eta \leq 1+y^{*}$, and two-sided otherwise.

For $\xi>n / 2$ consider $n-\xi$ and $1-\eta$ and make use of the relationships

$$
\theta_{L}(\xi, \eta, N, n, \gamma)+\theta_{U}(n-\xi-1,1-\eta, N, n, 2 \delta-\gamma)=1
$$

and

$$
\theta_{U}(\xi, \eta, N, n, \gamma)+\theta_{L}(n-\xi-1,1-\eta, N, n, \delta+\gamma)=1 .
$$

The generated value $y$ of $U(0,1)$ r.v. must be attached to the final report. Hence, now results are given by four numbers: population size, sample size, number of successes and the value $y$.

2. Because the population is finite, the set of admissible values of $\theta$ is also finite. For example, the standard confidence interval (for population of size 1000 ) when $x=2$ is observed is $[0.025,0.555]$. The parameter $\theta$ may take one of the values $\{0.025,0.026, \ldots, 0.554,0.555\}$ and the number of units with a given property is one of $\{25,26, \ldots, 554,555\}$.

3 . We now give an example of application. Let the size of a population be $N=1000$. We took a sample of size $n=100$ and we observed $\xi=2$ objects with a given property. Let the confidence level be $\delta=0.95$. The drawn value of the auxiliary variable $\eta$ is 0.2534 . The shortest confidence interval is $(0.0043349,0.0788678)$. Its length is 0.0745329 . 
The final report may look as follows:

$$
\begin{gathered}
N=1000 ; \quad n=100, \quad x=2, \quad y=0.2534, \quad \delta=0.95, \\
\theta \in(0.00433486 ; 0.0788678) .
\end{gathered}
$$

The standard confidence interval is $(0.006981,0.0824044)$ and its length equals 0.0754233 .

\section{References}

J. P. Buonaccorsi (1987), Note on confidence intervals for proportions in finite populations, Amer. Statistician 41, 215-218.

C. J. Clopper and E. S. Pearson (1934), The use of confidence or fiducial limits illustrated in the case of the binomial, Biometrika 26, 404-413.

W. G. Cochran (1977), Sampling Techniques, 3rd ed., Wiley.

H. Cramér (1946), Mathematical Methods in Statistics, Princeton Univ. Press (19th printing, 1999).

N. L. Johnson and S. Kotz (1969), Distributions in Statistics: Discrete Distributions, Houghton Mifflin, Boston.

E. L. Lehmann (1959), Testing Statistical Hypothesis, Springer (3rd ed., 2005).

J. Neyman (1934), On the two different aspects of the representative method: The method of stratified sampling and the method of purposive selection, J. Roy. Statist. Soc. 97, 558-625.

S. D. Silvey (1970), Statistical Inference, Chapman \& Hall (11th ed., 2003).

J. P. Wendell and J. Schmee (2001), Likelihood confidence intervals for proportions in finite populations, Amer. Statistician 55, 55-61.

T. Wright (1991), Exact Confidence Bounds when Sampling from Small Finite Universes; An Easy Reference Based on Hypergeometric Distribution, Lecture Notes in Statist. 66, Springer, New York.

F. Yates (1960), Sampling Methods for Censuses and Surveys, Hafner, New York.

W. Zieliński (2010a), The shortest Clopper-Pearson confidence interval for binomial probability, Comm. Statist. Simulation Comput. 39, 188-193.

W. Zieliński (2010b), Estimation of Proportion, Wydawnictwo SGGW, Warszawa (in Polish).

W. Zieliński (2011), Comparison of confidence intervals for fraction in finite populations, in: Quantitative Methods in Economics XII, SGGW, Warszawa, 177-182.

W. Zieliński (2012), The shortest confidence interval for probability of success in a negative binomial model, Appl. Math. (Warsaw) 39, 143-149.

W. Zieliński (2014), The shortest randomized confidence interval for probability of success in a negative binomial model, Appl. Math. (Warsaw) 41, 43-49.

W. Zieliński (2016), The shortest Clopper-Pearson randomized confidence interval for binomial probability, REVSTAT-Statist. J., to appear. 
Wojciech Zieliński

Department of Econometrics and Statistics

Warsaw University of Life Sciences

Nowoursynowska 159

02-776 Warszawa, Poland

E-mail: wojciech_zielinski@sggw.pl

http://wojtek.zielinski.statystyka.info 
